\title{
Evolución de la investigación y de la aplicación en Psicología del Deporte, a través del análisis de los Congresos Nacionales de Psicología del Deporte (1999-2008)
}

\author{
Progression of research and its application in Sport Psychology through the \\ analysis of the National Sport Psychology Congresses (1999-2008)
}

\author{
Aurelio Olmedilla ${ }^{1}$, Enrique Ortega ${ }^{1}$, Enrique J. Garcés de los Fayos ${ }^{2}$, \\ Pedro Jara ${ }^{2}$, Francisco J. Ortín ${ }^{2}$ \\ 1. Departamento de la Actividad Física y del Deporte. Universidad Católica San Antonio de Murcia \\ 2. Universidad de Murcia
}

CORRESPONDENCIA:

Aurelio Olmedilla Zafra

Universidad Católica San Antonio

Fac. de Ciencias de la Salud, de la Actividad Física y del Deporte

Campus de los Jerónimos, s/n.

30107 Guadalupe (Murcia)

aolmedilla@pdi.ucam.edu

\section{Resumen}

El presente trabajo analiza la evolución de la investigación y de la aplicación en Psicología del Deporte, a través de las aportaciones científicas presentadas en los congresos organizados por la Federación Española de Psicología del Deporte, durante la última década, desde 1999 hasta 2008. Se han analizado el total de aportaciones científicas $(n=436)$ registradas en los libros de actas de los cinco Congresos Nacionales de Psicología del Deporte: Murcia en 1999; Pontevedra en 2001; León en 2003; Málaga en 2005; y Sevilla en 2008. En ellos se han estudiado: a) el tipo de aportación científica; b) el área temática; c) el tipo de muestra utilizada; d) el tipo de deporte o ejercicio físico; e) los tipos de instrumentos utilizados; f) el número de referencias en idioma español; g) el número de referencias en otos idiomas; y h) el número de autores por trabajo.

Palabras clave: Psicología del deporte, congresos, investigación, aplicación.

\section{Abstract}

The present study analyzed the progression of Sport Psychology research and its application through the scientific contributions presented in the congresses organized by the Spanish Federation of Sport Psychology during the last decade, from 1999 through 2008. All scientific contributions $(n=436)$ registered in the proceedings of the five National Congresses of Sport Psychology were analyzed: Murcia in 1999, Pontevedra in 2001, León in 2003, Málaga in 2005, and Seville in 2008. The following aspects were studied: a) type of scientific contribution; b) subject matter; c) type of sample utilized; d) type of sport or exercise; e) types of instruments used; f) number of references in Spanish; g) number of references in other languages; and h) number of authors per study.

Key words: Sport psychology, congresses, research, application. 


\section{Introducción}

El papel que juega la producción científica en el seno de una sociedad es cada vez más valorado, de ahí que una buena parte de las instituciones contemple entre sus prioridades el apoyo de políticas para la potenciación y consolidación de grupos de investigación.

Dentro de las ciencias sociales, la psicología es un área de lento pero continuo crecimiento, y algunas disciplinas psicológicas, de más o menos reciente implantación, como la psicología del deporte, se encuentran en esta situación. El aumento de la población que practica ejercicio físico y deporte en nuestra sociedad es, quizá, una de las causas que permite este desarrollo. En este contexto, se observa, por un lado, una mayor productividad científica de los investigadores, y por otro, un creciente interés de los profesionales en publicar parte de sus trabajos aplicados. Los estudios bibliométricos permiten analizar el estado actual de la producción científica en un área determinada. En el área de las ciencias del deporte los criterios de análisis utilizados han sido, fundamentalmente, las revistas científicas (Valenciano, Antolín, Moreno, Devís y Villamón, 2003; Valenciano, Devís y Villalón, 2005, 2008) y los congresos (Pérez, 2007). En Psicología del Deporte se pueden encontrar estudios generales basados en el análisis de los congresos (Cantón y Sánchez-Gombau, 1999) o de las revistas (García-Mas, 1999; Quiñones, Garcés de los Fayos, Peñaranda, López y Jara, 2001), y estudios de carácter más específico, como el trabajo de García, Garcés de los Fayos y Jara (2005), sobre el papel de la atención en el ámbito deportivo.

Aunque la producción científica y la publicación de prácticas profesionales en el ámbito de la psicología del deporte ha sido objeto de algunos estudios más recientes (Garcés de Los Fayos, Vives y Dosil, 2004; Olmedi- lla y Ramírez, 2006), el desarrollo de la disciplina en estos últimos años hace que su contribución haya quedado superada por los acontecimientos, requiriendo un análisis actualizado de la realidad profesional de la psicología del deporte en España, tanto en su vertiente investigadora como en el ámbito de la aplicación.

Por ello se plantea este trabajo cuyo objetivo es describir la evolución de la investigación y de la aplicación en Psicología del Deporte, a través de los trabajos presentados en los Congresos Nacionales de Psicología de la Actividad Física y del Deporte, organizados por la Federación Española de Psicología del Deporte y las organizaciones autonómicas correspondientes.

\section{Metodología}

El universo de congresos nacionales de psicología del deporte organizados por la Federación Española de Psicología del Deporte está formado por un total de 11 congresos, desde el primero, realizado en el año 1992 en Sevilla, hasta el último, celebrado igualmente en Sevilla en el pasado 2008. Se ha seleccionado una muestra de los congresos realizados durante la última década, desde 1999 hasta 2008. Por un lado, por cuestiones de actualidad, y por otro, dado que ya existe un trabajo bibliométrico sobre los primeros congresos nacionales (Cantón y Sánchez-Gombau, 1999).

La recogida de datos se realizó a través de la consulta de las actas de cada uno de los congresos analizados. Se registraron 8 campos (ver tabla 1 ).

Se realizó un análisis descriptivo de cada una de las variables, registrando medias, desviación típica, mínimos y máximos para variables continuas, y recuentos y porcentajes para variables categóricas. Se utilizó el paquete estadístico SPSS 16.0.

Tabla 1. Criterios de recogida de datos de los congresos analizados

\begin{tabular}{|c|c|}
\hline 1. Tipo de aportación científica & 1) Conferencia; 2) Mesa Redonda; 3) Comunicación; 4) Simposium; 5) Póster; 6) Talleres \\
\hline 2. Área temática & $\begin{array}{l}\text { 1) Salud; 2) Entrenamiento psicológico; 3) Iniciación deportiva; 4) Gestión; 5) Arbitraje; } \\
\text { 6) Rendimiento deportivo; 7) Medios de comunicación; 8) Psicofisiología; 9) Otros }\end{array}$ \\
\hline 3. Tipo de muestra utilizada & $\begin{array}{l}\text { 1) Iniciación deportiva y deporte escolar; 2) Gestión; 3) Árbitros; 4) Alto Rendimiento y } \\
\text { profesionales; 5) Universitarios; 6) Adultos sedentarios; 7) Tercera edad; } \\
\text { 8) Deportistas adultos }\end{array}$ \\
\hline 4. Tipo de deporte o ejercicio físico & $\begin{array}{l}\text { 1) Deporte en equipo; 2) Deporte Individual; 3) Deporte en general; 4) Ejercicio físico; } \\
\text { 5) Otros }\end{array}$ \\
\hline 5. Tipos de instrumentos utilizados & 1) Cuestionario; 2) Hoja Observación; 3) Entrevista; 4) Experimental; 5) Test específicos \\
\hline \multicolumn{2}{|c|}{ 6. El número de referencias en idioma español } \\
\hline 7. El número de referencias en otros & iomas \\
\hline
\end{tabular}




\section{Resultados}

Los datos del presente estudio indican que en los últimos cinco Congresos Nacionales de Psicología del Deporte (1999-2008), se han realizado un total de 436 aportaciones científicas. En la figura 1 se aprecia el total de estas aportaciones, realizadas en cada uno de los congresos.

En la figura 2 se aprecia, del total de aportaciones científicas realizadas, el tipo de presentación. En concreto se observa que prácticamente la totalidad de aportaciones, o son comunicaciones, o son conferencias, o son póster (95,64\%). La gran mayoría son comunicaciones $(69,04 \%)$, seguidas por conferenciasponencias $(13,76 \%)$ y por póster científicos $(12,84 \%)$.

En la tabla 2 se muestra el tipo de presentación científica en los diferentes congresos objeto de estudio.

Los datos de la tabla 2 indican que el congreso en el que hubo mayor número de comunicaciones fue el de Murcia, seguido de Sevilla y León. Por otro lado, en el que hubo mayor número de conferencias-ponencias fue León, seguido de Málaga. En el congreso de Murcia destacaron las comunicaciones (96,84\%), en el de Pontevedra se aprecia un descenso importante en las aportaciones científicas, además de una mayor varia-

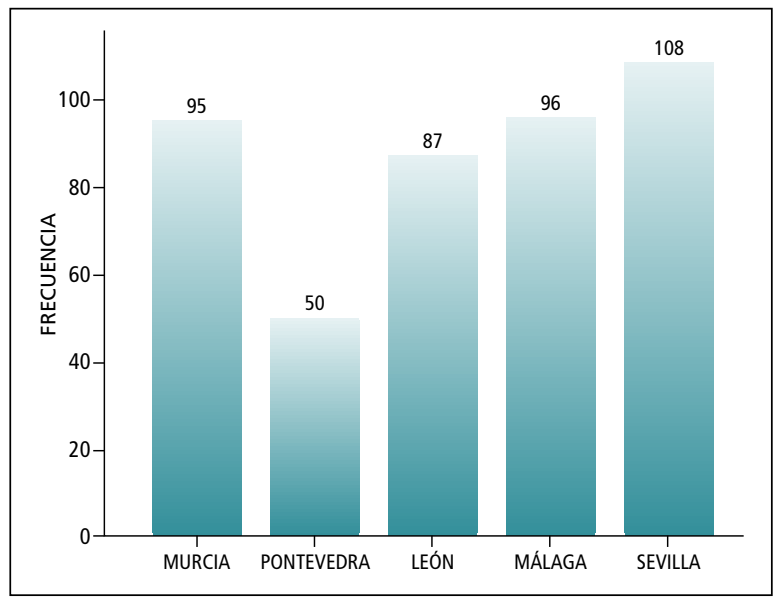

Figura 1. Aportaciones científicas realizadas en cada uno de los Congresos Nacionales de Psicología del Deporte (1999-2008) bilidad, en el de León destacan las conferencias, en el de Málaga se observa un descenso significativo de comunicaciones a expensas de incrementar los póster, y finalmente en el de Sevilla se vuelve a ver un incremento de comunicaciones, aunque muy alejado del de Murcia, y se mantienen como formato para la comunicación científica los póster, casi inexistentes en los tres primeros congresos.

En la tabla 3 se observa la distribución de las aportaciones científicas, según las áreas temáticas.

Como se observa en la tabla 3 , se aprecian cuatro áreas (motivación y emoción, entrenamiento psicológico, salud y calidad de vida, y modelos, metodología y evaluación) entre las cuales se distribuye el 54,61\% del total de las aportaciones científicas. Además de esas cuatro áreas temáticas que destacan en todos los congresos, en el de Murcia destaca también el área de Poblaciones especiales (14,70\%); en Pontevedra, la iniciación deportiva (10,20\%); en León, la Psicología organizacional y gestión (12,64\%); en Málaga, las nuevas tecnologías (13,82\%), y en Sevilla no se aprecia ningún área destacable fuera de las cuatro de la totalidad de congresos.

$\mathrm{Al}$ analizar, por un lado, la temática de las conferencias-ponencias, y por otro lado las de las comuni-

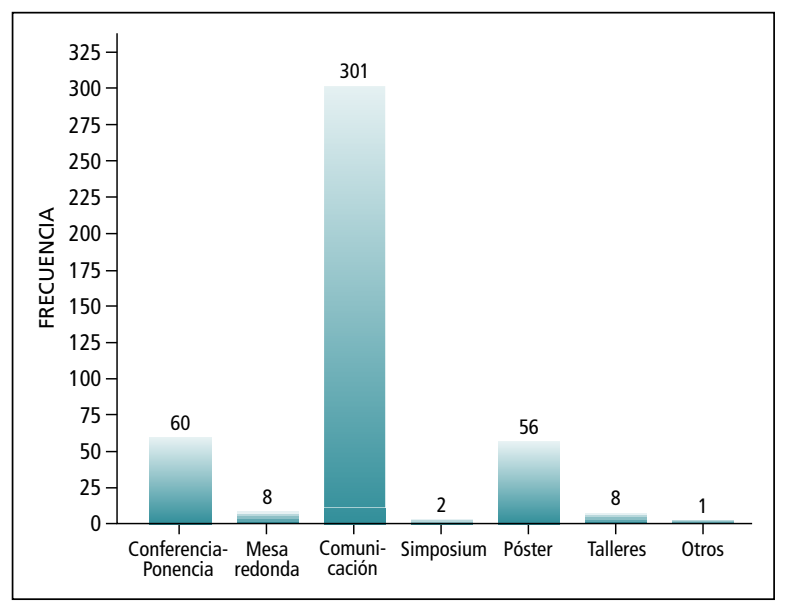

Figura 2. Aportaciones científicas realizadas en cada uno de los Congresos Nacionales de Psicología del Deporte (1999-2008), según el tipo de presentación científica.

Tabla 2. Tipo de presentación, según congreso

\begin{tabular}{|c|c|c|c|c|c|}
\hline & Murcia & Pontevedra & León & Málaga & Sevilla \\
\hline Conferencia-Ponencia & 2 & 11 & 26 & 19 & 2 \\
\hline Mesa Redonda & 0 & 0 & 0 & 0 & 8 \\
\hline Comunicación & 92 & 28 & 61 & 54 & 66 \\
\hline Simposium & 0 & 0 & 0 & 0 & 2 \\
\hline Póster & 0 & 11 & 0 & 23 & 22 \\
\hline Talleres & 0 & 0 & 0 & 0 & 8 \\
\hline Otros & 1 & 0 & 0 & 0 & 0 \\
\hline Total & 95 & 50 & 87 & 96 & 108 \\
\hline
\end{tabular}


Tabla 3. Aportaciones científicas según el área temática

\begin{tabular}{|c|c|c|c|c|c|c|}
\hline & Murcia & Pontevedra & León & Málaga & Sevilla & Total \\
\hline Motivación y emoción & 14 & 6 & 10 & 11 & 21 & 62 \\
\hline Entrenamiento psicológico & 9 & 8 & 12 & 8 & 20 & 57 \\
\hline Salud y calidad de vida & 8 & 7 & 9 & 17 & 12 & 53 \\
\hline Aprendizaje y desarrollo motor & 7 & 3 & 5 & 4 & 3 & 22 \\
\hline Poblaciones especiales & 14 & 3 & 3 & 7 & 2 & 29 \\
\hline Modelos, metodología y evaluación & 8 & 6 & 10 & 15 & 26 & 65 \\
\hline Iniciación deportiva & 13 & 5 & 6 & 7 & 4 & 35 \\
\hline Psicobiología del deporte & 9 & 3 & 3 & 3 & 1 & 19 \\
\hline Psicología organizacional y gestión & 7 & 1 & 11 & 7 & 2 & 28 \\
\hline Lesiones y retirada deportiva & 6 & 2 & 5 & 2 & 7 & 22 \\
\hline Arbitraje & 0 & 1 & 4 & 0 & 2 & 7 \\
\hline Nuevas tendencias & 0 & 5 & 9 & 13 & 8 & 35 \\
\hline
\end{tabular}

Tabla 4. Áreas temáticas según tipo de presentación científica

\begin{tabular}{|c|c|c|c|c|}
\hline & \multicolumn{2}{|c|}{ CONFERENCIA/PONENCIA } & \multicolumn{2}{|c|}{ COMUNICACIÓN/PÓSTER } \\
\hline & $\%$ de columna & Recuento & $\%$ de columna & Recuento \\
\hline Motivación y emoción & $13,33 \%$ & 8 & $14,37 \%$ & 51 \\
\hline Entrenamiento psicológico & $8,33 \%$ & 5 & $13,23 \%$ & 47 \\
\hline Salud y calidad de vida & $11,67 \%$ & 7 & $12,68 \%$ & 45 \\
\hline Aprendizaje y desarrollo motor & $5,00 \%$ & 3 & $5,35 \%$ & 19 \\
\hline Poblaciones especiales & $8,33 \%$ & 5 & $6,76 \%$ & 24 \\
\hline Modelos, metodología y evaluación & $20,00 \%$ & 12 & $13,24 \%$ & 47 \\
\hline Iniciación deportiva & $8,33 \%$ & 5 & $8,17 \%$ & 29 \\
\hline Psicobiología del deporte & $6,67 \%$ & 4 & $4,23 \%$ & 15 \\
\hline Psicología organizacional y gestión & $5,00 \%$ & 3 & $6,76 \%$ & 24 \\
\hline Lesiones y retirada deportiva & $5,00 \%$ & 3 & $5,35 \%$ & 19 \\
\hline Arbitraje & $3,33 \%$ & 2 & $1,13 \%$ & 4 \\
\hline Nuevas tendencias & $5,00 \%$ & 3 & $8,73 \%$ & 31 \\
\hline Total & $100 \%$ & 60 & $100 \%$ & 355 \\
\hline
\end{tabular}

cación-póster, en la tabla 4, se puede observar que, en ambos casos, las áreas temáticas que destacan son las relacionadas con modelos, metodología y evaluación, y motivación y emoción, si bien el orden se altera dependiendo de si son conferencias-ponencias o comunicaciones-póster.

Por otro lado, sí que se aprecia un mayor porcentaje de aportaciones científicas en las comunicacionespóster que en las conferencias-ponencias, en las áreas temáticas de entrenamiento psicológico y nuevas tendencias. Por el contrario, se aprecia un mayor porcentaje de aportaciones científicas en las conferencias-ponencias, que en las comunicaciones-póster en las áreas temáticas de modelos metodología y evaluación.

En la tabla 5 se muestran los datos relacionados con el tipo de muestra objeto de estudio de las diferentes aportaciones científicas.
En la tabla 5 se aprecia que desde una perspectiva del total de congresos objeto de estudio, el $29,77 \%$ de las aportaciones científicas realizan estudios con muestras de alto rendimiento, el 25,81\%, de iniciación y deporte escolar; el 6,74\%, de universitarios, y el 6,51\%, de deportistas adultos, apreciándose valores muy bajos en el resto de tipos de muestra. Por otro lado, la mayoría de estudios utiliza como muestra a deportistas de alto rendimiento, seguidos de aquellos cuya muestra son deportistas de iniciación. Además de estas dos muestras, en el congreso de Murcia destacan los estudios con árbitros, en Pontevedra y Málaga con universitarios, y en León y Sevilla con deportistas adultos.

En la tabla 6 se muestra el tipo de práctica deportiva o la realización de ejercicio físico. Se observa que el deporte en general y el deporte en equipo son las modalidades más estudiadas. De igual modo, destaca 
Tabla 5. Aportaciones científicas según la muestra objeto de estudio

\begin{tabular}{|c|c|c|c|c|c|c|}
\hline & Murcia & Pontevedra & León & Málaga & Sevilla & Total \\
\hline Iniciación deportiva & 25 & 9 & 21 & 22 & 34 & 111 \\
\hline Gestión & 4 & 0 & 0 & 2 & 3 & 9 \\
\hline Árbitros & 6 & 1 & 4 & 1 & 2 & 14 \\
\hline Alto rendimiento & 27 & 15 & 22 & 29 & 35 & 128 \\
\hline Entrenadores & 4 & 3 & 1 & 6 & 3 & 17 \\
\hline Universitarios & 4 & 4 & 7 & 9 & 5 & 29 \\
\hline Adultos sedentarios & 2 & 0 & 0 & 3 & 3 & 8 \\
\hline Deportistas adultos & 0 & 1 & 9 & 4 & 14 & 28 \\
\hline Tercera edad & 0 & 2 & 1 & 1 & 1 & 5 \\
\hline Otros & 23 & 15 & 22 & 17 & 4 & 81 \\
\hline
\end{tabular}

Tabla 6. Aportaciones científicas según la modalidad deportiva objeto de estudio

\begin{tabular}{|c|c|c|c|c|c|c|}
\hline & Murcia & Pontevedra & León & Málaga & Sevilla & Total \\
\hline Deporte en equipo & 22 & 17 & 27 & 19 & 29 & 114 \\
\hline Deporte individual & 15 & 7 & 19 & 20 & 11 & 72 \\
\hline Deporte en general & 23 & 18 & 24 & 30 & 44 & 139 \\
\hline Ejercicio físico & 4 & 4 & 2 & 13 & 16 & 39 \\
\hline Otros & 31 & 4 & 15 & 12 & 8 & 70 \\
\hline
\end{tabular}

Tabla 7. Aportaciones científicas según el instrumento utilizado

\begin{tabular}{|c|c|c|c|c|c|c|}
\hline & Murcia & Pontevedra & León & Málaga & Sevilla & Total \\
\hline Cuestionario & 43 & 15 & 43 & 34 & 42 & 177 \\
\hline Hoja de observación & 9 & 1 & 8 & 5 & 2 & 25 \\
\hline Entrevista & 8 & 2 & 5 & 4 & 5 & 24 \\
\hline Experimental & 6 & 0 & 0 & 11 & 9 & 26 \\
\hline Test específicos & 6 & 5 & 7 & 9 & 20 & 47 \\
\hline Otros & 23 & 27 & 23 & 10 & 30 & 113 \\
\hline
\end{tabular}

que apenas se aprecian diferencias en la distribución porcentual de los estudios según la modalidad deportiva en cada congreso, repitiéndose patrones semejantes en cada uno de ellos. Únicamente cabe destacar que conforme se ha evolucionado en el tiempo, el porcentaje de trabajos cuya muestra está formada por personas que realizan ejercicio físico, ha aumentado significativamente.

Igualmente, se puede apreciar que en el congreso celebrado en Murcia destacan otras modalidades deportivas, en Pontevedra, Málaga y Sevilla el deporte general, y en León el deporte en equipo.

En la tabla 7, se pueden observar las aportaciones científicas, según el tipo de instrumento utilizado en la investigación. Al analizar la totalidad de congresos, se observa que el instrumento más utilizado es el cuestionario, el cual es utilizado en el $42,96 \%$ de los trabajos de investigación. De igual modo destaca que es el más utilizado en todos los congresos, salvo en el congreso de Pontevedra, donde la categoría otros es superior $(54,1 \%$ frente a $30,2 \%)$

Tras el cuestionario y otros instrumentos, destaca el uso de tests específicos, los cuales también pueden ser considerados cuestionarios, con un uso en el $11,41 \%$ de los trabajos, destacando su uso en el congreso de Sevilla (18,52\% del total de ese congreso) y Málaga (12,33\% del total de ese congreso).

Por otro lado, el siguiente instrumento más utilizado son los propios de la metodología experimental, con un uso en el 6,31\% del total de trabajos analizados. En concreto el uso de este tipo de instrumento destaca en los congresos de Málaga (15,07\% del total de ese congreso) y de Sevilla (8,33\% del total de ese congreso).

Con respeto a la hoja de observación, propio de la metodología observacional, apenas es utilizada (6,07\% de los trabajos), destacando su uso en el congreso de Murcia (9,47\% del total de ese congreso) y en el de León (9,30\% del total de ese congreso). 
Tabla 8. Aportaciones científicas según el número de autores

\begin{tabular}{|c|c|c|c|c|c|c|}
\hline & Murcia & Pontevedra & León & Málaga & Sevilla & Total \\
\hline Uno & 25 & 17 & 27 & 22 & 29 & 120 \\
\hline Dos & 34 & 12 & 31 & 30 & 29 & 136 \\
\hline Tres & 21 & 10 & 13 & 29 & 20 & 93 \\
\hline Cuatro & 5 & 6 & 12 & 9 & 11 & 43 \\
\hline Cinco & 5 & 2 & 3 & 5 & 8 & 23 \\
\hline Seis & 1 & 1 & 0 & 1 & 4 & 7 \\
\hline Siete & 2 & 2 & 1 & 0 & 4 & 9 \\
\hline Ocho & 2 & 0 & 0 & 0 & 2 & 4 \\
\hline Nueve & 0 & 0 & 0 & 0 & 1 & 1 \\
\hline Media & 2,49 & 2,50 & 2,28 & 2,46 & 2,85 & 2,53 \\
\hline
\end{tabular}

Tabla 9. Media de referencias bibliográficas utilizadas en las aportaciones científicas presentadas a los Congresos Nacionales de Psicología del Deporte 1999-2008

\begin{tabular}{lcccccc}
\hline Referencias & Murcia & Pontevedra & León & Málaga & Sevilla & Total \\
\hline Castellano & $7,4 \pm 11,8$ & $5,7 \pm 6,6$ & $7,0 \pm 7,1$ & $7,3 \pm 6,6$ & $5,1 \pm 4,7$ & $6,5 \pm 7,9$ \\
\hline Otros idiomas & $8,8 \pm 12,5$ & $5,8 \pm 7,1$ & $8,6 \pm 9,8$ & $12,8 \pm 10,8$ & $11,8 \pm 14,5$ & $9,9 \pm 11,9$ \\
\hline Totales & $16,3 \pm 22.3$ & $11,5 \pm 11,3$ & $15,7 \pm 11,6$ & $20,1 \pm 12,6$ & $17,0 \pm 15,1$ & $16,4 \pm 15,8$ \\
\hline
\end{tabular}

Finalmente, cabe destacar el escaso uso de las entrevistas (un 5,83\% del total de los trabajos). En concreto, destaca el uso de la entrevista en el congreso de Murcia, con un $8,42 \%$ del total de trabajos realizados en dicho congreso.

En la tabla 8, se aprecia el número de aportaciones científicas según el número de autores firmantes en cada una de ellas. En concreto, del total de aportaciones científicas analizadas, aparece una media de 2,53 autores por trabajo, apreciándose un mínimo de 1 y un máximo de 9 . La mayoría de aportaciones científicas están firmadas por dos autores (31,19\%), seguidas de las firmadas por un solo autor (27,52\%).

$\mathrm{Al}$ analizar cada uno de los congresos, se observa que en el congreso celebrado en Murcia destacan los trabajos firmados por dos autores (35,79\%), seguidos de trabajos firmados por un solo autor (26,32\%); en Pontevedra, por un autor (34,1\%), seguido de dos autores $(24,1 \%)$; en León, los firmados por dos autores $(35,63 \%)$ seguido de los firmados por un solo autor (31,03\%); en Málaga, los firmados por dos autores $(31,25 \%)$ seguido de los firmados por tres autores $(30,21 \%)$; y en Sevilla, los firmados por uno y dos autores tienen el mismo porcentaje (26,85\%).

En la tabla 9 se aprecian las medias de referencias bibliográficas utilizadas por las aportaciones científicas objeto de estudio, registrando una media aproximada de 17 referencias por trabajo, con un mínimo de cero y un máximo de 65 . En concreto, se observa una tendencia a la reducción de referencias en castellano y al incre- mento en otros idiomas. De igual modo, cabe destacar que en todos los congresos el número medio de referencias bibliográficas en castellano es inferior al de referencias en otros idiomas, fundamentalmente en inglés.

\section{Discusión}

Pararse a observar cómo se encuentra la disciplina en la que los investigadores trabajan es un ejercicio necesario, sobre todo si se quiere consolidar los conocimientos que ya tienen un desarrollo específico, así como si se desea insertar, en el cuerpo de conocimientos, nuevos constructos y teorías que ayuden a conocer mejor los diferentes ámbitos de una ciencia. Con ese objetivo se ha realizado este trabajo y para ello se ha realizado una aproximación a una realidad concreta: ¿cuál ha sido la evolución de la última década en Psicología del Deporte?, y por tanto ¿cuál es su situación actual? Para ello, se ha procurado establecer una continuación lógica sobre dos trabajos anteriores (Quiñones et al., 2001; Garcés de los Fayos et al., 2004), que pueden aportar algunos datos relevantes sobre lo que ya se pudo observar en los mismos.

Por otra parte, y complementariamente a los trabajos citados, se ha pretendido mejorar la perspectiva al intentar completar, con datos sobre los congresos nacionales, lo que se pudo observar en el análisis pertinente de las dos principales revistas científicas de psicología del deporte que se editan en España, y que sí se 
había tenido en cuenta en dichos trabajos. A partir de ahí, los resultados que se han mostrado en el apartado anterior ofrecen algunas tendencias que sugieren reflexionar acerca del presente y, sobre todo, del futuro de la psicología del deporte en nuestro país.

Así, en primer lugar, en los tres últimos congresos el aumento en la presentación de trabajos científicos es un hecho incuestionable, lo cual incide en varias reflexiones a considerar: el hecho de que se está ante una disciplina claramente dinámica, con mayor número de grupos de investigación y, lo más importante, con una cantidad más amplia de líneas de investigación que permiten observar cómo la ciencia va consolidándose, al tiempo que ampliando sus miras e intereses científicos. Concretamente, se podrían destacar tres tipos de orientaciones dentro de la psicología del deporte, que pueden ayudar a comprender cuál puede ser el futuro que esta disciplina va a recorrer en los próximos años:

- Por un lado, las áreas que se podrían denominar "clásicas": motivación y emoción (un área quizás ya en franca decadencia si se atiende al hecho de que las aportaciones innovadoras son cada vez menores), entrenamiento psicológico (área que sigue manteniendo su papel preponderante, ya que aglutina a los psicólogos del deporte que también trabajan directamente con deportistas, y que sigue siendo un área trascendental; en este sentido, sería de agradecer que las revistas científicas del área destinaran alguna sección específica para que los psicólogos pudieran comunicar sus trabajos de intervención y entrenamiento psicológico; en la actualidad sólo la Revista de Psicología del Deporte recoge este aspecto de manera estructural; quizá por la escasez de medios en los que publicar, o por la propia inercia de los psicólogos más alejados del ámbito académico a no hacerlo, se echa en falta una mayor aportación, y por lo tanto un mayor grado de innovación en las propuestas; la revista Cuadernos de Psicología del Deporte dedicó dos monográficos a trabajos aplicados en el ámbito del fútbol, con algunas experiencias muy interesantes (Almeida, 2004; Andreu, 2003; De la Vega, R., 2004; Valiente, Pérez-Córdoba, Gamito, Gómez, Sánchez y Morilla, 2003; Vives y Garcés de Los Fayos, 2003), salud y calidad de vida (que va aglutinando un número de trabajos significativos, pero que quizás debería empezar a proponer líneas de investigación más específicas que se perciben como de gran interés, como por ejemplo el amplio espectro de aplicaciones a través de la actividad física y deportiva en enfermedades físicas concretas) y modelos, metodología y evaluación (que siempre estará presente, en tanto que supone los aspectos básicos y técnicos en los que se suelen apoyar el resto de áreas de investigación).

- Luego estarían las áreas influenciadas por variables coyunturales, muy ligadas a momentos concretos como, por ejemplo, el estudio de poblaciones especiales, la iniciación deportiva o la psicología organizacional y gestión deportiva. Cada una de ellas se caracteriza por tener un desarrollo irregular, coincidente con varios grupos de investigación que, en un periodo concreto, suelen manifestar un mayor grado de interés en las mismas, lo que supone que a veces estén muy presentes y otras, en cambio, casi desaparezcan, no apreciándose líneas de investigación continuadas, salvo excepciones muy concretas. Quizás, la iniciación deportiva, como área de trabajo específica, pueda estar a medio camino entre este grupo de áreas y el anterior.

- Finalmente, aquellas áreas aún por desarrollar. Suele tratarse de temáticas muy concretas, que al no ser investigadas por varios grupos de trabajo o, al menos, uno con potencial evidente, no terminan de aparecer trabajos que se desarrollen con el paso del tiempo, por lo que siempre aparece como el grupo de temas que son innovadores porque escasas veces se atiende como cuerpo de conocimiento por el que "apostar" en investigación. Así, se puede citar, entre otras, los aspectos psicobiológicos en el deporte, las lesiones y sus correlatos mentales, o la casuística psicológica del arbitraje. Sin embargo, es necesario destacar algunos hechos que permiten albergar esperanzas de consolidación de alguna de estas áreas. En este sentido, y respecto al área de estudio de las lesiones deportivas y psicología, en estos últimos años se ha ido formando un Grupo de Investigación específico de este ámbito en la Universidad Católica San Antonio de Murcia, que cuenta con doctores, no sólo en psicología, sino en medicina y en ciencias del deporte, de diferentes universidades españolas y portuguesas, que ha permitido conseguir diferentes Proyectos de Investigación, así como la organización recientemente de un Simposium específico de lesiones y psicología, tratado de manera multidisciplinar; además de la publicación continuada de trabajos (Abenza, Olmedilla, Ortega y Esparza, en prensa; Olmedilla, García-Montalvo y MartínezSánchez, 2006; Olmedilla, Andreu, Abenza, Ortín y Blas, 2006; Olmedilla, Andreu, Ortín y Blas, 2008; Olmedilla, Andreu, Ortín y Blas, en prensa; Olmedilla, Ortega, Abenza y Esparza, en prensa) y de la lectura de tesis de máster (Prieto, 2008) y DEA (Abenza, 2007) y de tesis doctorales (Ortín, 2009). También es de destacar la, cada vez, mayor producción de trabajos en el ámbito de la psicología y los 
árbitros, sobre todo las publicaciones provenientes de la Universidad de las Palmas de Gran Canaria (Betancor, 1998; Cabrera, Ruiz y Marrero, 1998; Guillén, 2003; Guillén, 2006; Marrero y Gutiérrez, 2006) y de la Universidad del País Vasco (AlonsoArbiol, Arratibel y Gómez, 2008; Alonso-Arbiol, Falcó, López, Ordaz y Ramírez, 2005; Ramírez, Alonso-Arbiol, Falcó y López, 2006).

Un segundo aspecto haría referencia a la población objeto de estudio en los trabajos científicos. Como se ha podido observar, principalmente son los deportistas de alto rendimiento y de iniciación en los que se aglutina la mayor parte de trabajos. Quizá este hecho no sólo es la realidad actual, sino que debería seguir siéndolo en el futuro porque, en definitiva, siguen siendo los dos grandes contextos donde el psicólogo del deporte desarrolla su actividad profesional. Preocupa, no obstante, el hecho de que los trabajos donde las muestras sean de entrenadores o de árbitros sigan siendo escasas. Pareciera que el deporte con el resto de "figuras" que lo constituyen no es todavía objetivo científico de los psicólogos del deporte y esto, como parece lógico, puede supone un lastre importante para el desarrollo científico de la disciplina.

Por último, y en tercer lugar, indicar que se ha descrito la estructura técnica de los trabajos científicos que últimamente se vienen desarrollando en psicología del deporte. Así, se puede comprobar que en cuanto al tipo de instrumentos de evaluación que se han manejado en los estudios analizados, lo mayoritario sigue siendo el uso de cuestionarios y autoinformes, "los instrumentos validados", cuantificables y/o experimentales que parecen dotar de mayor "tranquilidad" al investigador.

La pregunta sería ¿dónde queda la investigación cualitativa, la investigación ecológica y, en definitiva, los instrumentos subjetivos? Salvando honrosas excepciones (Escudero, Balagué y García-Mas, 2002; Sánchez y Torregrosa, 2005) la producción científica en este sentido no es muy boyante. Las normas establecidas en la "competición investigadora", en la que la comunidad científica y académica se encuentra inmersa, obliga a los investigadores a trabajar fundamentalmente con este tipo de instrumentos, en trabajos técnicos que han quedado perfectamente reflejados en los datos más específicos de este estudio, referidos a referencias bibliográficas utilizadas o números de firmantes por trabajo, por citar sólo algún ejemplo. No obstante, quizás será conveniente reflexionar acerca de la necesidad futura de abordar los trabajos cualitativos que, en muchas ocasiones, reflejan con mayor coherencia la realidad deportiva que tanto interesa a la psicología del deporte. 
Abenza, L. (2007). Análisis de la respuesta psicológica en la rehabilitación y adherencia de los deportistas lesionados. DEA: Universidad Católica San Antonio de Murcia.

Abenza, L., Olmedilla, A., Ortega, E. y Esparza, F. (en prensa). Estados de ánimo y adherencia a la rehabilitación de deportistas lesionados. Apunts, Medicina de l'Esport.

Almeida, P. (2004). Intervençao psicologica no futebol: reflexoes de uma experiencia com uma equipa da liga portuguesa profissional. Cuadernos de Psicología del Deporte, 4(1-2), 181-190.

Alonso-Arbiol, I., Arratibel, N. y Gómez, E. (2008). La motivación del colectivo arbitral en fútbol: un estudio cualitativo. Revista de Psicología del Deporte, 17(2), 187-203.

Alonso-Arbiol, I., Falcó, F., López, M., Ordaz, B. y Ramírez, A. (2005). Development of a questionnaire for the assessment of sources of stress in Spanish soccer referees. Ansiedad y Estrés, 11(2-3), 175188.

Andreu, M.D. (2003). Programa de entrenamiento psicológico y metodología de trabajo en un equipo de fútbol juvenil. Cuadernos de Psicología del Deporte, 3(2), 83-95.

Betancor, M.A. (1998). Bases para la formación del árbitro de baloncesto. Tesis Doctoral: Universidad de Las Palmas de Gran Canaria.

Cabrera, D., Ruiz, G. y Marrero, G. (1998). El perfil de las jueces de gimnasia rítmica. Las Palmas de Gran Canaria: Lecarez.

Cantón, E. y Sánchez Gombáu, M.C. (1999). Los Congresos Nacionales de Psicología del Deporte a través del análisis de sus actas. En G. Jiménez y E.J. Garcés de los Fayos (Eds.), La Psicología del Deporte en España. 1999. Murcia: Sociedad Murciana de Psicología de la Actividad Física y el Deporte.

De la Vega, R. (2004). Pilares básicos de la intervención psicológica realizada en el fútbol profesional. Cuadernos de Psicología del Deporte, 4(1-2), 159-180.

Garcés de los Fayos, E.J., Vives, L. y Dosil, J. (2004). Nuevas aportaciones en Psicología del Deporte. Una mirada crítica sobre la última década de la disciplina en España. Cuadernos de Psicología del Deporte, 4(1-2), 7-18.

García, J., Garcés de los Fayos, E.J. y Jara, P. (2005). El papel de la atención en el ámbito deportivo: Una aproximación bibliométrica de la literatura recogida en la base de datos Psyclit. Revista de Psicología del Deporte, 14(1), 125-140.

García-Mas, A. (1999). La Revista de Psicología del Deporte 19891999: Una historia de diez años de encuentros. En G. Jiménez y E.J. Garcés de los Fayos (Eds.), La Psicología del Deporte en España 1999. Murcia: Sociedad Murciana de Psicología de la Actividad Física y el Deporte.

Guillén, F. (2003). Psicología del arbitraje y el juicio deportivo. Barcelona: INDE.

Guillén, F. (2006). Psicología del arbitraje y del juicio deportivo. En E.J. Garcés de los Fayos, A. Olmedilla y P. Jara (coords.), Psicología y deporte, (pp. 667-684). Murcia: Diego Marín.

Marrero, G. y Gutiérrez, C. (2002). Las motivaciones de los árbitros de fútbol. Revista de Psicología del Deporte, 11, 69-82.

Olmedilla, A. y Ramírez, P. (2006). Aplicación de la psicología en el ámbito de la actividad física y el deporte. En E.J. Garcés de los Fa- yos, A. Olmedilla y P. Jara (coords.), Psicología y deporte, (pp. 237258). Murcia: Diego Marín.

Olmedilla, A., Andreu, M.D., Abenza, L., Ortín, F.J. y Blas, A. (2006). Lesiones y factores deportivos en futbolistas jóvenes. Cultura, Ciencia y Deporte, 2(5), 59-66.

Olmedilla, A., Andreu, M.D., Ortín, F.J. y Blas, A. (2008). Epidemiología lesional en futbolistas jóvenes. Cultura, Ciencia y Deporte, 3(9), 64-77.

Olmedilla, A., Andreu, M.D., Ortín, F.J. y Blas, A. (en prensa). Ansiedad competitiva, percepción de éxito y lesiones: un estudio en futbolistas. Revista Internacional de Medicina de Ciencias de la Actividad Física y el Deporte.

Olmedilla, A., García-Montalvo, C. y Martínez-Sánchez, F. (2006). Factores psicológicos y vulnerabilidad a las lesiones deportivas: un estudio en futbolistas Revista de Psicología del Deporte, 15(1), 37-52.

Olmedilla, A., Ortega, E., Abenza, L. y Esparza, F. (en prensa). Lesiones y factores psicológicos en futbolistas jóvenes. Archivos de Medicina del Deporte.

Ortín, F.J. (2009). Factores psicológicos y sociodeportivos y lesiones en jugadores de fútbol semiprofesionales y profesionales. Tesis Doctoral: Universidad de Murcia.

Pérez, V. (2007). Análisis de las contribuciones a los congresos del área de Didáctica de la Expresión Corporal (1990-2000). Apunts. Educación Física y Deportes, 87, 27-34.

Prieto, J.M. (2008). Relación entre factores físico-deportivos y psicológicos con la incidencia de lesiones deportivas en tenistas. Tesis de Master: Universidad Católica San Antonio de Murcia.

Quiñones, E., Garcés de los Fayos, E.J., Peñaranda, M., López, J.J. y Jara, V. (2001). Un acercamiento a la comprensión de la psicología del deporte en España. Análisis de la Revista de Psicología del Deporte 1992-1999. Revista de Psicología del Deporte, 10(1), 69-81.

Ramírez, A., Alonso-Arbiol, I., Falcó, F. y López, M. (2006). Programa de intervención psicológica con árbitros de fútbol. Revista de Psicología del Deporte, 15(2), 311-325.

Valenciano, J., Antolín, L., Moreno, A., Devís, J. y Villamón, M. (2003). Las revistas científico-técnicas españolas de las Ciencias de la Actividad Física y el Deporte: Inventario y análisis de calidad de contenido y difusión, Revista española de documentación científica, 26(2), 177-190.

Valenciano, J., Devís, J. y Villamón, M. (2005). Análisis de la visibilidad de las revistas científico-técnicas españolas de Ciencias de la Actividad Física y el Deporte. Revista de Psicología del Deporte, 14(2), 253-267.

Valenciano, J., Devís, J. y Villamón, M. (2008). Análisis comparativo de la calidad de las revistas científico-técnicas españolas de Ciencias de la Actividad Física y el Deporte. Information Research, 13(1), 337.

Valiente, M., Pérez-Córdoba, E., Gamito, J.M., Gómez, M.A., Sánchez, E. y Morilla, M. (2003). Planificación psicológica de la cantera del Sevilla F.C. S.A.D.: organización, funcionamiento y programa deportivo-formativo. Cuadernos de Psicología del Deporte, 3(2), 17-34.

Vives, L. y Garcés de Los Fayos, E.J. (2003). Intervención psicológica en un club de fútbol base: propuesta de un sistema de actuación psicológica desde sus diversas áreas. Cuadernos de Psicología del Deporte, 3(2), 51-66. 